\title{
Experiencia en sedoanalgesia vinculada a procedimientos en un departamento de emergencia pediátrica
}

\author{
Mariana Más Gómez*, Paloma Amarillo Martínez†, María Soledad Tórtorał, \\ Patricia Dall'Orso§, Javier Prego Petit
}

\section{Resumen}

Introducción: la realización de procedimientos dolorosos es cada vez más frecuente en el área de urgencias pediátricas. El control del dolor, mitigar el temor y la ansiedad es una pieza clave en la atención del paciente pediátrico y una de las prioridades del médico emergencista.

Objetivo: describir la experiencia en sedoanalgesia con ketamina y fentanilo como único fármaco o asociados a otros para procedimientos dolorosos en el Departamento de Emergencia Pediátrica (DEP) del Centro Hospitalario Pereira Rossell (CHPR).

Material y método: estudio descriptivo, retrospectivo. Período: enero de 2011 a julio de 2016. Población: todos los pacientes que recibieron sedoanalgesia con ketamina o fentanilo (solos o asociado a otros fármacos) para realización de procedimientos dolorosos en el DEP-CHPR. Base de datos: historias clínicas. Se midió la eficacia mediante el éxito del procedimiento y la seguridad por la presencia de efectos adversos vinculados a la misma.

Resultados: $n=352$, menores de 15 años; 96 recibieron ketamina y 256 recibieron fentanilo. Media de edad: 7 años, sexo masculino: 245. Dosis media de ketamina $1 \mathrm{mg} / \mathrm{kg}$. Dosis media de fentanilo $1 \mathrm{\gamma} / \mathrm{kg}$. Principales indicaciones de sedoanalgesia: procedimientos ortopédicos (264), toracocentesis (62). Procedimiento con éxito: 352 pacientes. Monitorización cardiovascular y saturometría durante el procedimiento: 100\%. Efectos adversos: seis (no requiriendo maniobras de soporte vital avanzado). Ningún paciente cambió su destino final.

Conclusiones: la utilización de sedoanalgesia resultó eficaz y segura realizada por el pediatra emergencista capacitado en el manejo farmacológico y de soporte vital avanzado. La monitorización del procedimiento durante y luego de éste es necesaria para pesquisar y resolver precozmente las complicaciones.

Palabras clave: Manejo del dolor

Analgesia

Servicio de urgencia en hospital

Niño

Analgésicos

Ketamina

Fentanilo
Key words: $\quad$ Pain management

Analgesia

Emergency service, hospital

Child

Analgesics

Ketamine

Fentanyl

\footnotetext{
* Prof. Adj. Emergencia Pediátrica. Facultad de Medicina, Universidad de la República. Departamento de Emergencia Pediátrica. Hospital Pediátrico - Centro Hospitalario Pereira Rossell.

† Asist. Emergencia Pediátrica. Facultad de Medicina, Universidad de la República. Departamento de Emergencia Pediátrica. Hospital Pediátrico - Centro Hospitalario Pereira Rossell.

‡ Prof. Adj. Emergencia Pediátrica. Facultad de Medicina, Universidad de la República. Departamento de Emergencia Pediátrica. Hospital Pediátrico - Centro Hospitalario Pereira Rossell.

§ Prof. Agda. Emergencia Pediátrica. Facultad de Medicina, Universidad de la República. Departamento de Emergencia Pediátrica. Hospital Pediátrico - Centro Hospitalario Pereira Rossell.

II Prof. Emergencia Pediátrica. Facultad de Medicina, Universidad de la República. Departamento de Emergencia Pediátrica. Hospital Pediátrico Centro Hospitalario Pereira Rossell.

Hospital Pediátrico. Centro Hospitalario Pereira Rossell

Correspondencia: Dra. Mariana Más. Correo electrónico: marianamas@gmail.com

Recibido: 17/2/20

Aprobado: 10/8/20
} 


\section{Introducción}

La realización de procedimientos dolorosos es frecuente en los servicios de urgencia pediátrica. Las técnicas de sedoanalgesia tienen el objetivo de controlar el dolor y la ansiedad, evitar los movimientos del paciente y proporcionar cierto grado de pérdida de memoria del procedimiento a fin de permitir un proceso con éxito y seguridad, minimizando las respuestas psicológicas desfavorables, asociadas a intervenciones médicas doloro$\operatorname{sas}^{(1-5)}$.

El adecuado control del dolor en el paciente pediátrico es un pilar fundamental en la asistencia que comenzó a tenerse en cuenta a partir de la década de $1980^{(6)}$. En 1985, la Academia Americana de Pediatría (AAP) y la Academia Americana de Odontólogos Pediátricos (AAPD) publicaron la primera serie de guías para la monitorización y manejo de los niños que requerían sedación para procedimientos electivos fuera del block quirúrgico ${ }^{(7)}$. Desde entonces el número de trabajos y publicaciones en relación con la realización de sedoanalgesia en la edad pediátrica en los departamentos de emergencia se ha incrementado exponencialmente ${ }^{(2-4,8-10)}$.

Existen diferentes protocolos para la sedoanalgesia en procedimientos considerados muy dolorosos realizados en área de urgencia. Durante el período del estudio en el Departamento de Emergencia Pediátrica del Centro Hospitalario Pereira Rossell (DEP-CHPR) la asociación de fármacos más utilizada fue fentanilo-midazolam y ketamina-midazolam- atropina ${ }^{(11,12)}$. En Uruguay, no todos los centros que asisten a niños y adolescentes disponen de protocolos de sedoanalgesia diseñados para cada procedimiento, lo cual constituye un importante desafío $^{(2-4,8-11)}$.

\section{Objetivo}

Describir la experiencia en sedoanalgesia con ketamina y fentanilo en procedimientos dolorosos, en el DEPCHPR.

\section{Población, material y método}

Se realizó un estudio descriptivo, retrospectivo, en el DEP-CHPR, en el período comprendido entre enero de 2011 a julio de 2016. Fueron enrolados todos los pacientes menores de 15 años, en quienes se utilizó la asociación de ketamina o fentanilo (solos o asociados a otros fármacos) para la realización de procedimientos dolorosos.

Se utilizó como fuente de datos las historias clínicas.

Las variables que se analizaron fueron: sexo, edad, procedimiento realizado, vía de administración, asociación farmacológica utilizada, dosis del fármaco usado, monitoreo del paciente, la presencia de complicaciones
Tabla 1. Características de la población. DEP. HP CHPR. 2011-2016.

\begin{tabular}{lc}
\hline Edad & Rango: 9 meses - 14 años. \\
Sexo (fi) & Media : 7 años \\
& F: 107 \\
Ketamina (fi) & 96 \\
Fentanilo (fi) & 256 \\
Éxito en el procedimiento (fi) & 350 \\
Complicaciones (fi) & 6 \\
Admisiones Hospitalarias (fi) & 141 \\
\hline (fi): frecuencia absoluta &
\end{tabular}

y el destino del usuario una vez finalizado el procedimiento.

En todos los pacientes se realizó monitorización cardiovascular y saturometría de pulso.

Se contó con aprobacióndel Comité de Ética del CHPR.

\section{Resultados}

Se enrolaron un total de 352 pacientes en el período comprendido del estudio. Las características de la población y los procedimientos realizados se muestran en las tablas 1 y 2 , respectivamente. Se colocó acceso vascular para realizar sedoanalgesia en el DEP a 255 pacientes, de éstos, 76 lo requerían para el tratamiento de la enfermedad que motivó la consulta. Los 97 pacientes restantes arribaron al DEP con acceso vascular periférico colocado por decisión del médico que realizó el traslado.

La dosis de ketamina utilizada estuvo comprendida en un rango de $0,5-2,5 \mathrm{mg} / \mathrm{kg}$, con una media $1 \mathrm{mg} / \mathrm{kg}$, y la dosis de fentanilo utilizada comprendía un rango entre $0,5-3 \gamma / \mathrm{kg}$, con una media de $1 \gamma / \mathrm{kg}$.

La elección de la asociación de fármacos para sedoanalgesia en cada procedimiento estuvo a cargo del pediatra de guardia capacitado en el uso de estos fármacos y soporte vital avanzado (tabla 3 ).

El procedimiento pudo ser realizado con éxito en 352 pacientes; en dos niños la toracocentesis fue en blanco.

En cuanto al registro de complicaciones, se muestra en la tabla 4. Se destaca que el episodio de depresión neuropsíquica se presentó en un niño de 3 años de edad, en quien se realizó reducción de fractura, con la asociación de fentanilo y midazolam. Requirió ventilación transitoria con bolsa máscara, con buena resolución, no 
Tabla 2. Procedimientos realizados con sedoanalgesia. DEP. HP - CHPR. 2011-2016.

\begin{tabular}{lccc}
\hline Procedimientos & Ketamina (fi) & Fentanilo (fi) & Total (fi) \\
\hline Ortopédicos & 34 & 234 & 268 \\
Toracocentesis & 56 & 6 & 62 \\
Punción lumbar & 3 & 1 & 4 \\
Drenaje absceso & & 2 & 2 \\
Reducción de hernia inguinal & 1 & 9 & 1 \\
Punción diagnóstica ósea o articular & & 1 & 9 \\
Extracción de cuerpo extraño & 2 & 3 & 3 \\
Exploración y sutura de heridas & & 256 & 352 \\
Total & 96 & & 3
\end{tabular}

(fi): frecuencia absoluta

Tabla 3. Fármacos utilizados para sedoanalgesia. DEP. HP-CHPR. 2011-2016.

\begin{tabular}{lc}
\hline Fármacos & (fi) \\
\hline Ketamina & 17 \\
Ketamina + Midazolam + Atropina & 41 \\
Ketamina + Fentanilo + Midazolam & 1 \\
Ketamina + Midazolam & 21 \\
Ketamina + Atropina & 16 \\
Fentanilo + Midazolam & 255 \\
Fentanilo + Dipirona & 1 \\
\hline
\end{tabular}

(fi): frecuencia absoluta

presentó otras complicaciones, ni requirió admisión hospitalaria.

En ningún caso se requirió progresar con soporte vital avanzado, ni modificar el destino del paciente vinculado al procedimiento, ni a la sedoanalgesia utilizada.

Los pacientes que recibieron fentanilo solo o asociado a otros fármacos permanecieron en el DEP menos de 2 horas luego del procedimiento. Los pacientes que recibieron ketamina sola o asociada a otros fármacos tuvieron un promedio de estadía en DEP de 5 horas luego del procedimiento.

En 141 casos se indicó admisión hospitalaria, vinculada a la enfermedad de base del niño y no debida a complicaciones del procedimiento. A 200 pacientes se les
Tabla 4. Complicaciones. DEP. HP-CHPR. 2011-2016.

\begin{tabular}{lcl}
\hline Complicaciones & (fi) & Fármacos utilizados \\
\hline Vómitos & 1 & Ketamina \\
& 1 & Ketamina + Midazolam \\
Broncoobstrucción & 1 & Fentanilo + Midazolam \\
Depresión neuropsíquica & 1 & Fentanilo + Midazolam \\
Taquicardia + Hipotensión & 1 & Ketamina + Atropina \\
Tos + Desaturación & 1 & Fentanilo + Midazolam \\
\hline (fi): frecuencia absoluta & & \\
\hline
\end{tabular}

otorgó el alta hospitalaria desde el servicio de urgencia. En 11, no se cuenta con el dato.

\section{Discusión}

Una debilidad de los estudios retrospectivos es la posible pérdida de datos, en este caso el correcto registro de las historias clínicas permitió acceder de manera confiable a los datos analizados.

Se destaca el alto porcentaje de éxito en el número de maniobras. En el período de estudio las asociaciones de fármacos utilizados fue la recomendada en el DEP-CHPR, basadas en la experiencia nacional e internacional, si bien no existían protocolos formales establecidos $^{(2-4,8-12)}$.

La dosis de fármacos utilizada concuerda con las recomendaciones. Hubo pacientes que requirieron dosis 
sucesivas, comprendidas en el límite superior, para lograr el efecto deseado. Comenzar con dosis bajas titulando las mismas, respetando el tiempo de inicio de acción hasta lograr al efecto deseado y utilizar escalas de sedación y analgesia, es lo recomendado para alcanzar el objetivo terapéutico con un menor riesgo de efectos ad$\operatorname{versos}^{(13-16)}$.

Contar con protocolos para procedimientos de sedoanalgesia en los departamentos de emergencia y personal entrenado es esencial para brindar una mejor asistencia al paciente y su familia desde la institución, constituyendo uno de los estándares de calidad ${ }^{(10,14-17)}$.

En esta cohorte de pacientes se requirió colocar acceso vascular a $51 \%$ de los pacientes únicamente para realizar sedoanalgesia. Este dato es de importancia para considerar en el futuro vías alternativas, como la yugal o intranasal, y evitar la punción con aguja cuando no es necesario ${ }^{(18-21)}$.

Uno de los aspectos más importantes para garantizar la seguridad del paciente, sobre todo en los casos que se administran fármacos por vía endovenosa, es realizar una planificación previa que incluye la evaluación del candidato a la sedoanalgesia. La adecuada monitorización durante la realización de los procedimientos para pesquisar precozmente los efectos adversos, como se realizó en esta cohorte, y contar con recursos humanos capacitados para conocer y manejar las posibles complicaciones es fundamental cuando se implementa este tipo de procedimientos en un servicio ${ }^{(14-18)}$.

$\mathrm{Al}$ igual que en otras publicaciones, el porcentaje de complicaciones fue bajo $(1,7 \%)$, ninguno de ellos requirió soporte vital avanzado. Una limitante es no contar en este período de estudio con capnógrafo, que alerta más precozmente las complicaciones respiratorias por hipoventilación en relación con la saturometría de pulso, siendo la presencia de hipoxemia un elemento más tardío ${ }^{(14,22-24)}$.

Las horas de estadía en DEP para los pacientes que recibieron ketamina fueron más prolongadas que las de los que recibieron fentanilo. En el subgrupo de pacientes que recibieron ketamina hubo más necesidad de admisión hospitalaria debido a la patología de base que motivó la consulta. Esto podría explicar la estadía más prolongada en el DEP. No obstante, si consideramos al subgrupo de pacientes ambulatorios (n 207), el $80 \%$ de los que recibieron fentanilo fueron dados de alta en un lapso $\leq 2$ horas y $60 \%$ de los que recibieron ketamina, presentando una diferencia significativa $(\mathrm{p} \leq 0,05)$. A todos ellos se les pudo otorgar el alta desde el servicio de emergencia en condiciones seguras. Este factor podría considerarse en el momento de elegir la asociación de fármacos a utilizar.

\section{Conclusiones}

Si bien se trata de un estudio retrospectivo, el número de pacientes enrolados justifica considerar sus resultados como base para futuras investigaciones e intervenciones.

La realización de procedimientos dolorosos en la urgencia puede realizarse de manera segura, eficaz y sin dolor utilizando fármacos y personal entrenado.

Se propone establecer protocolos de sedoanalgesia para procedimientos en los servicios de urgencia brindando al equipo de salud referencias para una actuación equitativa y segura para los pacientes. Proporcionar a la familia información por escrito para el seguimiento del paciente luego del egreso permitiría pesquisar las complicaciones tardías.

\section{Summary}

Introduction: painful procedures are gradually increasing frequency in pediatrics emergency units. Controlling pain and mitigating fear and anxiety are of the essence when assisting pediatric patients and one of the priorities of emergency doctors.

Objective: to describe using sedoanalgesia with ketamine and fentanyl as the only drug or associated with other drugs during painful procedures, at the Pediatrics Emergency Unit, of the Pereira Rossell Hospital Center.

Method: descriptive, retrospective study from January 2011 to July 2016. Population: all patients who received sedoanalgesia with ketamine and fentanyl (as the only drug or associated with other drugs) during painful procedures at the Pediatrics Emergency Unit, of the Pereira Rossell Hospital Center. Database: medical records. Effectiveness was measured by success of the procedure and safety in terms of adverse effects in connection with it.

Results: $\mathrm{N}=352$ children younger than 15 years old. 96 received ketamine and 256 received fentanyl. Average age was 7 years old and 245 of them were boys. Average dose of ketamine was $1 \mathrm{mg} / \mathrm{kg}$, average dose of fentanyl was $1 \gamma / \mathrm{kg}$. Main indications for sedoanalgesia included: orthopaedic procedures (264), thoracentesis (62). Procedures were successful in 352 patients. 100\% of cases involved cardiovascular monitoring and blood oxygen saturation meter. Adverse effects: 6 (no advanced life support manoeuvres required). No patient changed final destination.

Conclusions: the use of sedoanalgesia was effective and safe, when applied by emergengy pediatricians qualified in the handling of drugs and advanced life support. Monitoring during and after procedure is required to determine and solve complications at an early stage. 


\section{Resumo}

Introdução: a realização de procedimentos dolorosos é cada vez mais frequente na área de emergência pediátrica. Controlar a dor, mitigar o medo e a ansiedade é um elemento-chave no atendimento ao paciente pediátrico e uma das prioridades do médico de emergência.

Objetivo: descrever a experiência em sedação e analgesia com ketamina e fentanil como fármaco único ou associado a outros para procedimentos dolorosos, no Serviço de Emergência Pediátrica (DEP) do Centro Hospitalar Pereira Rossell (CHPR)

Material e métodos: estudo descritivo retrospectivo. Período: janeiro de 2011 a julho de 2016. População: todos os pacientes que receberam sedação e analgesia com ketamina ou fentanil (isoladamente ou em combinação com outras drogas) para realização de procedimentos dolorosos no DEP-CHPR. Banco de dados: prontuários médicos. A eficácia foi medida pelo sucesso do procedimento e a segurança pela presença de efeitos adversos associados.

Resultados: $\mathrm{n}=352$ crianças menores de 15 anos. 96 receberam ketamina e 256 receberam fentanil. Idade média: 7 anos, sexo masculino: 245. Dose média de ketamina $1 \mathrm{mg} / \mathrm{kg}$. Dose média de fentanil $1 \gamma / \mathrm{kg}$. Principais indicações para sedação e analgesia: procedimentos ortopédicos (264), toracocentese (62). Procedimento com sucesso: 352 pacientes. Monitorização cardiovascular e oximetria durante o procedimento: 100\%. Efeitos adversos: 6 (não requer manobras de suporte avançado de vida). Nenhum paciente mudou seu destino final.

Conclusões: o uso da sedação e analgesia foi eficaz e seguro realizado por pediatra de emergência capacitado em manejo farmacológico e suporte avançado de vida. O monitoramento do procedimento durante e após é necessário para investigar e resolver precocemente as complicações.

\section{Bibliografía}

1. Alonso MT. Introducción. En: Capapé S, edit. Manual de analgesia y sedación en urgencias de pediatría. $2^{\mathrm{a}}$ ed. Madrid: Ergon, 2012:1-8.

2. Krauss B, Green SM. Procedural sedation and analgesia in children. Lancet 2006; 367(9512):766-80. Disponible en: www.thelancet.com [Consulta: 2 noviembre 2019]. doi:10.1016/S0140-6736(06)68230-5.

3. Hartling L, Milne A, Foisy M, Lang E, Sinclair D, Klassen T, et al. What works and what's safe in pediatric emergency procedural sedation: an overview of reviews. Acad Emerg Med 2016; 23:519-30.

4. Doctor K, Roback M, Teach S. An update on pediatric hospital-based sedation. Curr Opin Pediatr 2013, 25:310-6. doi:10.1097/MOP.0b013e328360bb92.
5. López JC, Soult JA. Analgesia y sedación en Pediatría. Pediatr Int 2006; 10(4):267-76.

6. Anand KJ, Hickey PR. Pain and its effects in the human neonate and fetus. N Eng1 J Med 1987; 317:1321-9.

7. Lowrie L. Evolution of a medical discipline during a professional lifetime. En: Gooden C, Lowrie L, Jackson B, edits. The pediatric procedural sedation handbook. Londres: Oxford University Press, 2018:3-8.

8. Capapé S, Benito FJ. Analgesia y sedación para procedimientos menores. En: Capapé S, edit. Manual de analgesia y sedación en urgencias de pediatría. $2^{\mathrm{a}}$ ed. Madrid: Ergon, 2012:175-82.

9. Sacchetti A, Cortese T. Procedual sedation and analgesia. En: Fuchs S, Yamamoto L, edits. APLS: the pediatric emergency medicine resource. $5^{\text {th }}$ ed. Miami: Jones and Bartlett Learning, 2012.

10. Mason K, Seth $\mathbf{N}$. The pearls of pediatric sedation: polish the old and embrace the new. Minerva Anestesiol 2019; 85(10):1105-17. doi: 10.23736/S0375-9393.19.13547-X.

11. Cardozo A. Dolor y ansiedad. Analgesia y sedación en la emergencia. En: Bello O, Sehabiague G, Prego J, de Leonardis D. Pediatría: urgencias y emergencias. $3^{\mathrm{a}}$ ed. Montevideo: BiblioMédica, 2009:207-22.

12. de Leonardis D, Prego J, Bello O, Pujadas M. Analgesia y sedación en procedimientos ortopédicos dolorosos en la sala de urgencias pediátricas. Arch Pediatr Urug 2006; 77(4): 361-7.

13. Fernaìndez Santervas Y, Gonzaìlez Herrero M, Duraìn Fernaìndez-Feijoo C. Analgesia sistémica. En: Capapé S, edit. Manual de analgesia y sedación en urgencias de pediatría. $2^{\text {a }}$ ed. Madrid: Ergon, 2012:87-99.

14. Adriaìn Gutièrrez $\mathbf{J}$, Miìguez Navarro $\mathbf{C}$. Complicaciones de los procedimientos de sedacioìn y analgesia en urgencias pediaitricas. En: Capapé S, edit. Manual de analgesia y sedación en urgencias de pediatría. $2^{\mathrm{a}}$ ed. Madrid: Ergon, 2012: 61-70.

15. Green S, Roback M, Krauss B, Miner J, Schneider S, Kivela $\mathbf{P}$, et al. Unscheduled procedural sedation: a multidisciplinary consensus practice guideline. Ann Emerg Med 2019; 73(5):e51-e65.

16. Hartling L, Milne A, Foisy M, Lang E, Sinclair D, Klassen T, et al. What works and what's safe in pediatric emergency procedural sedation: an overview of reviews. Acad Emerg Med 2016: 23(5):519-30.

17. Coté CJ, Wilson S. Guidelines for monitoring and management of pediatric patients before, during, and after sedation for diagnostic and therapeutic procedures. Pediatrics 2019; 143(6):e20191000.

18. Trottier E, Ali S, Le May S, Gravel J. Treating and reducing anxiety and pain in the paediatric emergency department: the TRAPPED survey. Paediatr Child Health 2015; 20(5):239-44.

19. Clerigué N. Analgesia y sedación para procedimientos mayores dolorosos. En: Capapé S, edit. Manual de analgesia y 
sedación en urgencias de pediatría. $2^{\mathrm{a}}$ ed. Madrid: Ergon, 2012:183-200.

20. Tsze DS, Reynolds J. Intranasal medications. En: Gooden C, Lowrie L, Jackson B, edits. The pediatric procedural sedation handbook. Londres: Oxford University Press, 2018.

21. Therapeutic intranasal drug delivery. Needleless treatment options for medical problems. Disponible en: www.intranasal.net [Consulta: 24 setiembre 2019].

22. Thackeray EM, Johnson KB. Airway rescue during sedation: a proposed airway rescue pathway for nonanesthesiologists. 2019; 32(4):464-71.
23. Grunwell JR, Travers C, McCracken CE, Scherrer PD, Stormorken AG, Chumpitazi CE, et al. Procedural sedation outside of the operating room using ketamine in 22,645 children: a report from the pediatric sedation research consortium. Pediatr Crit Care Med 2016; 17(12):1109-16.

24. Bellolio MF, Puls HA, Anderson JL, Gilani Wi, Murad MH, Barrionuevo $\mathbf{P}$, et al. Incidence of adverse events in paediatric procedural sedation in the emergency department: a systematic review and meta-analysis. BMJ Open 2016; 6:e011384. doi:10.1136/bmjopen-2016-011384.

\footnotetext{
Contribución de autores

Todos los autores participaron en igual medida en las distintas etapas de elaboración del artículo.

Javier Prego, https://orcid.org/0000-0002-6568-6547

Patricia Dall'Orso, https://orcid.org/0000-0002-2526-7575

Mariana Más, https://orcid.org/0000-0001-8531-6810

Soledad Tórtora, https://orcid.org/0000-0001-7182-8030

Paloma Amarillo, https://orcid.org/0000-0002-4677-5747
} 